\title{
Quasiclassical states for the Coulomb problem and the dynamical algebra so $(4,2)$
}

\section{DAVID S. MCANALLY}

The Coulomb problem is one of the most important and most studied problems in quantum mechanics. Nevertheless, some aspects of the problem have remained only partially explored.

For example, the time-dependence does not seem to have been determined explicitly for any nonconstant observable of this system in the Heisenberg picture. One result of the present work is the use of the 'dynamical group (and algebra)' approach to identify observables for the Coulomb problem with a simple time-dependence.

Another aspect of quantum mechanics which has received considerable attention in recent years is the study of coherent states, which are generalisations (in various directions) of special states of the simple harmonic oscillator, first constructed by Schrödinger in 1926. These oscillator states behave 'quasiclassically', in that the expectation values of the coordinate and momentum operators obey the coresponding classical equations of motion, while the uncertainties do not increase in time.

There have been several efforts to construct analogous quasiclassical states for the Coulomb problem, but these previous approaches can all be criticised on various grounds. The major result of the present work is the construction of a set of quasiclassical states for the 3-dimensional Coulomb problem, using the dynamical algebra approach, and the investigation of their time evolution. These states are defined as initially coherent states (in the Barut-Girardello sense) of a dynamical so(4,2) Lie algebra of natural variables for the problem. (These are the variables mentioned above, that have a simple time-dependence in the Heisenberg picture.) The initial states so defined are then allowed to evolve under the action of the Coulomb Hamiltonian, in order to determine quasiclassical states at later times. The ensuing rates of spread of these states, measured in terms of the natural variables, are investigated in detail in near-ionization limit (that is, states with $E \lesssim 0$ where $E$ is a characteristic value of the energy).

\section{Received 4th June, 1990.}

Thesis submitted to the University of Queensland, June 1989. Degree approved January 1990. Supervisor Dr A.J. Bracken.

Copyright Clearance Centre, Inc. Serial-fee code: 0004-9729/90 \$A2.00+0.00. 
In Chapter 1, the standard features of the coherent states of the simple harmonic oscillator (Schrödinger's states) are reviewed, and two specific generalisations are investigated: the coherent states defined by Perelomov (a Lie group-theoretic definition) and those defined by Barut and Girardello (a Lie algebra-theoretic definition). The details of both sorts of states for the discrete series of representations of the Lie algebra so $(2,1)$ are reproduced from the respective papers, and a connection found between them. The representation of so $(4,2)$ appropriate for the Coulomb problem is then investigated in a new oscillator realisation, convenient for what follows; a rather elementary Perelomov state is found, and the general Barut-Girardello state is constructed. Coherent angular momentum states for the two-dimensional isotropic harmonic oscillator are constructed, by diagonalising lowering operators for the absolute value of the angular momentum and for the radial quantum number. These states are shown to differ from the usual coherent states, defined with respect to the coordinate vector and conjugate momentum, but they also behave quasiclassically. This illustrates the important point that the algebraic construction of quasiclassical states can be dependent on the choice of dynamical algebra for the problem.

In the second chapter, a 'tilted' so $(4,2)$ dynamical algebra is introduced for the Coulomb problem, and is shown to provide a natural set of quantum variables for the system, with simple time-dependence in the Heisenberg picture. The very complicated functional forms of the operators in terms of the coordinate vector $\underset{\sim}{\boldsymbol{r}}$ and momentum vector $\boldsymbol{p}$ are derived, so that their classical analogues can be found and interpreted geometrically in Chapter 3. The simple time-dependence of the operators becomes a periodic time-dependence in the classical limit, and this is shown to reflect a simple functional dependence of the tilted classical variables on action-angle variables for the problem.

In order to test and illustrate techniques to be applied to the 3-dimensional problem, the simpler case of a 1-dimensional quantum Coulomb problem, formulated in terms of an so(2,1) dynamical algebra, is considered in Chapter 4 . The corresponding coherent states of Barut and Girardello and of Perelomov are simple to construct and describe in this case, having been studied by those authors.

In the fifth and final chapter, the main problem is treated. The $\mathrm{SO}(4,2)$ states of Perelomov, defined with respect to the natural so $(4,2)$ operators for the 3-dimensional Coulomb problem, are shown in general not to evolve quasiclassically in any reasonable sense. On the other hand, the so $(4,2)$ states of Barut and Girardello, defined with respect to the same natural operators, are shown to evolve in a quasiclassical way in the near-ionization region, at least for times of the order of the classical period $\tau$. It is also shown that in this region the uncertainties in the nonconstant $\operatorname{so}(4,2)$ operators increase over times of order $\tau^{7 / 6}$, and that at regular intervals of order $\tau^{4 / 3}$, these 
uncertainties actually decrease temporarily.

Comparison is made between the results obtained here and those of earlier attempts in the literature to construct quasiclassical states for this sytem.

\section{Department of Mathematics}

The University of Queensland

Queensland 4072

Australia 